\title{
Mapping the Stability Diagram of a Digital Ion Trap (DIT) Mass Spectrometer Varying the Duty Cycle of the Trapping Rectangular Waveform
}

\author{
Alberto Berton, ${ }^{\text {P Pietro Traldi, }}{ }^{\text {a }}$ Li Ding, $^{\text {b }}$ and Francesco L. Brancia ${ }^{\text {b }}$ \\ ${ }^{a}$ National Research Council-Institute of Molecular Science and Technologies (CNR-ISTM), Padova, Italy \\ ${ }^{\mathrm{b}}$ Shimadzu Research Laboratory (Europe), Manchester, United Kingdom
}

In a digital ion trap the $\beta_{r}$ and $\beta_{z}$ boundary lines of the stability diagram are determined experimentally using an innovative approach. In the rectangular waveform-driven digital ion trap (DIT) manipulation of the waveform duty cycle allows introduction of a precisely defined DC quadrupole component into the main trapping field. Variation of the duty cycle can be controlled at software level without any hardware modification. The data generated use peptide ions, which produce stability diagrams in good agreement with the theoretical stability diagrams previously determined by simulation studies. (J Am Soc Mass Spectrom 2008, 19, 620-625) @ 2008 American Society for Mass Spectrometry

$\mathrm{T}$ The intrinsic simplicity of the three-dimensional Paul ion trap combined with its small dimension (consisting of two end caps and one intermediate ring electrode arranged in a cylindrical symmetry) contributed to its commercial success as a bench-top instrument [1,2]. Nevertheless, optimization of its analytical performance has required the combined efforts of several research groups across the world [3]. In particular, determination of ion motion in the trap proved to be crucial in understanding the factors that influence mass resolution and mass accuracy.

Ion motion in a quadrupole field generated inside an ion trap by application of a $U+V \cos (\Omega t)$ voltage at the ring electrode can be easily described by the stability diagram related to the $a$ and $q$ variables defined as

$$
\begin{aligned}
& q_{u}=\frac{4 z V}{m r^{2} \Omega^{2}} \\
& a_{u}=-\frac{8 z V}{m r^{2} \Omega^{2}}
\end{aligned}
$$

where $m$ and $z$ are the mass and the charge of the ions; $r$ is the equatorial radius of the trap (in Paul ion trap $\left.r^{2}=2 z^{2}\right) ; U$ is the DC component of the voltage applied to the ring electrode; $V$ is the amplitude of the radio frequency $(\mathrm{RF})$ voltage applied to the ring electrode; and $\Omega$ is the RF frequency.

Address reprint requests to Dr. Francesco Brancia, Shimadzu Research Laboratory (Europe), Biological Mass Spectrometry, Wharfside Trafford Wharf Road, Manchester M17 1GP United Kingdom. E-mail: francesco.brancia@ srlab.co.uk
It is important to point out that Eqs. 1 and 2 contain all six parameters affecting ion motion inside the trap. Since the interdependence of these six parameters would require a six-dimensional space, a bidimensional space defined by $a$ and $q$ is commonly used. The stability diagram represents the portion of $a, q$ space in which ions follow stable trajectories inside the trap. When the $a, q$ values of an ion confined in a particular ion trap geometry that has specific voltages applied to it that lie outside the stability diagram, ion motion becomes unstable and the ion is ejected. Since their commercialization ion traps have suffered from the limited mass resolution. The low-resolution originates mainly from two different and unwanted phenomena. The first is related to space charge effects: if the ions inside the trap are too high in number, an additional DC component is produced. To correctly describe ion motion the vectorial sum of the main RF field with that generated by the ion cloud needs to be taken into account [4]. In practice, the correct ion motion diverges far from the theoretical conditions and ion injection into the trap has to be carefully monitored and regulated, so that the trap is not overfilled with ions [5, 6]. The second phenomenon that affects mass resolution is the scanning speed used. It has been shown that a significant reduction in the scanning speed can lead to mass resolution close to $10^{6}$ [7]. This interesting feature is not present in the Paul ion traps that are commercially available when analysis is carried out over the entire mass range, although it is possible for a limited mass range (zoom scan).

A new trapping device has recently been designed, based on a completely novel approach. Its development started from the consideration that, instead of using 
sinusoidal waveforms, rectangular waveforms could be used to drive the ion trap $[8,9]$. Under these conditions quadrupole trapping and excitation waveforms are generated by the rapid switching between discrete DC voltage levels. The switching timing of the rectangular waveforms can be easily and precisely controlled. The device produced, called digital ion trap (DIT), allows mass spectra to be obtained by means of a frequency scan instead of the conventional voltage scan and, consequently, a wider mass range of analysis is observed [10-12]. Rectangular waveforms can be manipulated by the control software so that a well-defined DC component can be superimposed on the main trapping field by variation of the waveform duty cycle. Herein this unique feature is exploited to map the stability diagram of the digital ion trap using peptide ions.

\section{Experimental}

\section{Materials}

All chemicals were analytical grade. Formic acid (FA), bradykinin fragment 1-5 (97\%, HPLC grade), and leukine enkephalin (96\%) were supplied by Sigma (Poole, UK). The organic solvents methanol, acetonitrile $(\mathrm{ACN})$, and water were obtained from Rathburn (Walkerburn, Scotland).

\section{Mass Spectrometry}

All spectra were acquired on a prototype DIT mass spectrometer based on the design of an LCMS 2010 single quadrupole mass spectrometer (Shimadzu Corporation, Kyoto, Japan) fitted with an electrospray probe. A sample was dissolved in 50\% (vol/vol) acetonitrile acidified with $0.1 \%$ (vol/vol) FA. Peptides were sprayed at flow rates of $10 \mu \mathrm{L} / \mathrm{min}$ with a concentration of $5 \mathrm{pmol} / \mu \mathrm{L}$. Ion source, curved desolvation line (CDL), and transfer ion optics were similar to those used on the commercial single quadrupole instrument. All lenses were optimized for the maximum transmission of the ions. The DC voltage applied to the external field-adjusting electrode positioned next to the entrance aperture was held at $500 \mathrm{~V}$. Helium leaked into the trap through a needle valve was used as buffer gas at an estimated pressure of $2.5 \mathrm{mTorr}$. During the frequency scan, the voltage on the ring electrode was held constant at an amplitude of $500 \mathrm{~V}$. All ions were recorded on an electron multiplier detector (ETP, Sydney, Australia). The ion signal was digitized by a DAQ NI-6111 board (National Instruments, Austin, TX, USA), using a Pentium III computer. Spectra were the sum of at least 50 scans each. Data acquisition was performed using custom-built software. In the instrument the frequency scan rate was designed so that a linear relationship between scan time and mass-to-charge $(\mathrm{m} / \mathrm{z})$ ratio is satisfied. Therefore, by using an external calibration it is possible to convert ejection time into $\mathrm{m} / \mathrm{z}$ ratio. Spectra were processed in our custom-built software. Ion abun- dance was varied using different introduction times. In the case of bradykinin fragment $1-5$ the introduction time was $30 \mathrm{~ms}$ and for leukine enkephalin was $40 \mathrm{~ms}$. Ions were cooled for $30 \mathrm{~ms}$ using helium. To experimentally determine the boundaries of the stability diagram, an in-house duty cycle calculator application was written. Thus, for a given $m / z$ value, trapping potential, and duty cycle the software is able to calculate the period for which the ion is theoretically brought into resonance at the boundary of the stability region. The period so determined is then entered in the scan table and used to acquire a mass spectrum. Before resonant ejection the ions are maintained for $40 \mathrm{~ms}$ at the boundaries using the period provided by the duty cycle calculator. If the experiment is in agreement with the theory using this period a depletion of the ion under investigation (normally under $50 \%$ of the original ion abundance) is expected to occur since ions cross the $\beta_{r}=0$ and $\beta_{z}=1$ lines. Using this approach, several duty cycle values were used to generate sufficient $a, q$ points for mapping the $\beta_{r}$ and $\beta_{z}$ boundaries.

\section{Results}

The fundamental theory of operation of quadrupole devices driven by a rectangular or pulsed wave voltage has been described extensively elsewhere [9-13]. In the DIT mass spectrometer, the trapping electric field and resonant excitation are provided by high-voltage switching circuits. In the steady trapping operation, a periodic rectangular wave voltage generated by switching between a high-voltage level $V_{1}$ and a low-voltage level $V_{2}$ is applied to the ring electrode of the ion trap. Under these conditions the Mathieu equation cannot be used to determine ion motion in the DIT and the matrix transform method must be used $[8,13]$. However, the Mathieu parameters $a$ and $q$ (see Eqs 1 and 2) can still be used to describe the DIT theoretical stability diagram. In the case of the DIT the $U$ and $V$ values are considered to be the average values of the DC and AC components of the rectangular wave voltage applied to the intermediate electrode, defined as [8]

$$
\begin{aligned}
& \mathrm{U}=\mathrm{d} \mathrm{V}_{1}+(1-\mathrm{d}) \mathrm{V}_{2} \\
& \mathrm{~V}=2\left(\mathrm{~V}_{1}-\mathrm{V}_{2}\right)(1-\mathrm{d}) \mathrm{d}
\end{aligned}
$$

As shown in Figure 1 the duty cycle is described as the ratio between $\tau$ and the total period $T$ of the rectangular wave. Unlike the sinusoidal wave, the rectangular wave can be generated with different pulsing times for $V_{1}$ and $V_{2}$ produced by the digital circuitry. In a digital ion trap the $a$ value is a function of both the DC offset and the duty cycle $d$. In fact, the DC component $U$ can be generated either by an imbalance between $V_{1}$ and $V_{2}$ or by variation of the duty cycle $d$. For mapping the stability diagrams, the values of the switching voltage levels $V_{1}$ and $V_{2}$ are kept at -500 and $500 \mathrm{~V}$, respec- 


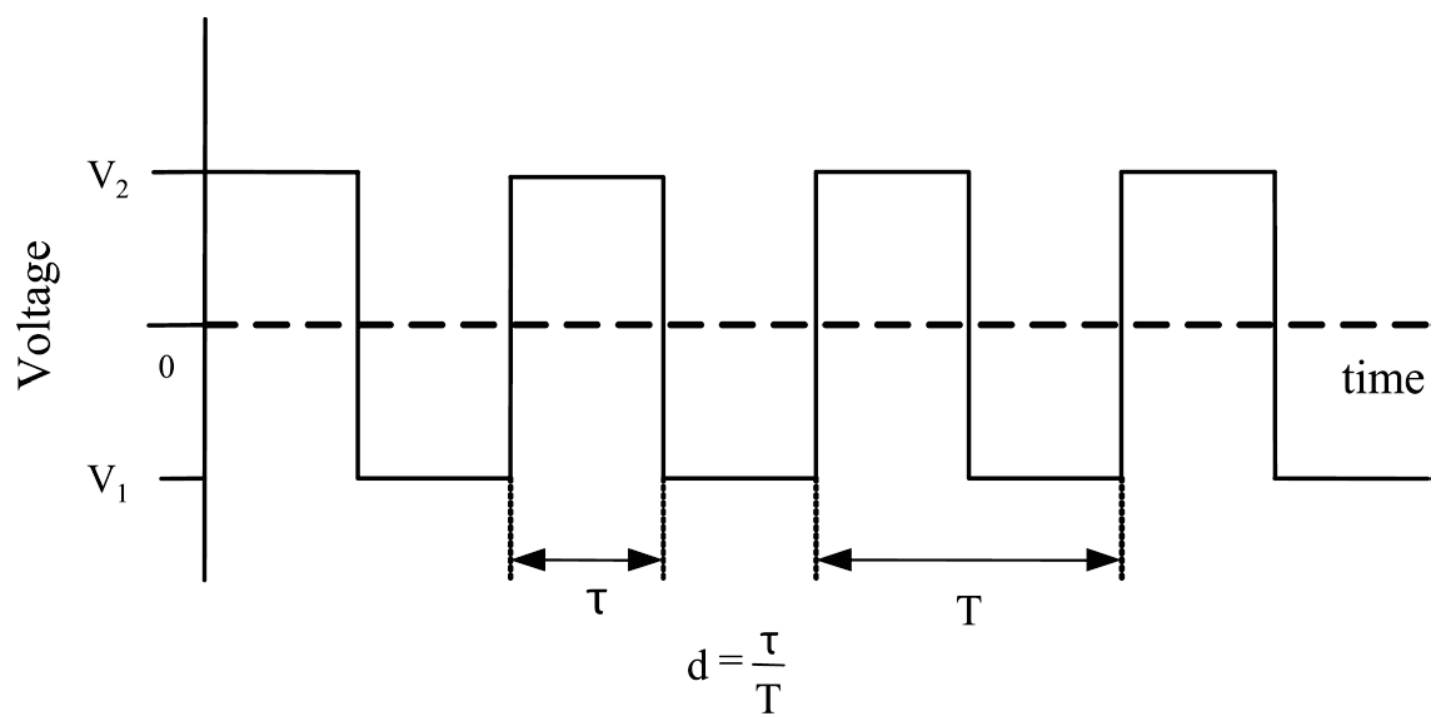

Figure 1. Schematic representation of the rectangular waveform of RF drive potential. $d$ represents the duty cycle for a given period $T$.

tively, so that the DC offset is 0 and only the duty cycle is changed to determine the stability boundaries.

When the duty cycle is $50 \%$, the DC quadrupole component is absent and ion motion can be described using only the $q$ axis. In the DIT the normal ejection point when mass analysis is performed using mass- selective instability scan mode is $q_{z}=0.712$ for $a_{z}=0$ and $\beta_{z}=1$. However, with a duty cycle diverging from $50 \%$ the DC quadrupole component generated has to be taken into account. Under these conditions, a new straight line passing through the origin of the $a / q$ axis has to be considered in accordance with the duty cycle

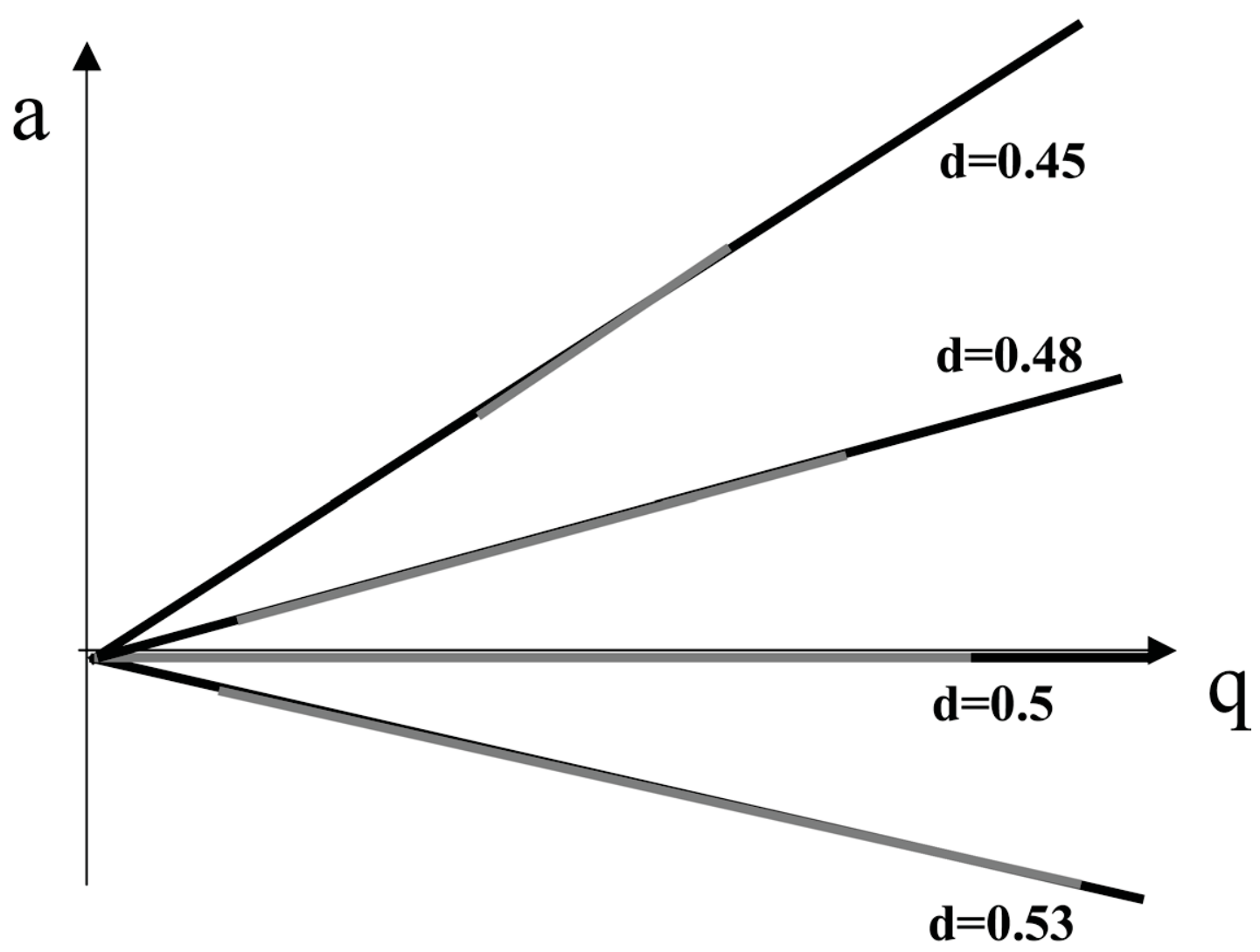

Figure 2. Various $a / q$ lines obtained by modulation of the rectangular waveform duty cycle. The gray part indicates the region of stability for a given duty cycle. 


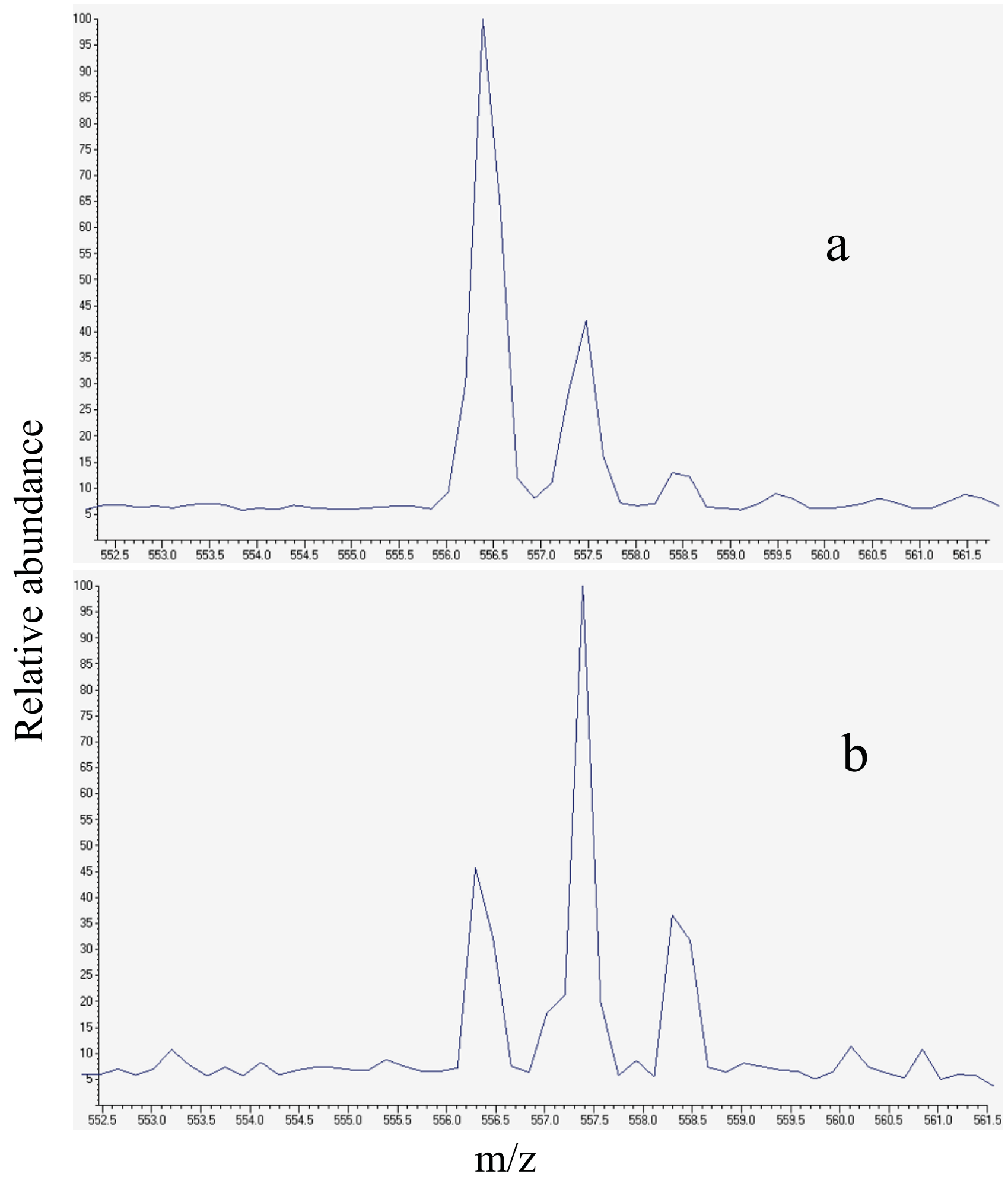

Figure 3. Leukine enkephalin $[\mathrm{M}+\mathrm{H}]^{+}$spectra: (a) inside the stability diagram and (b) on the boundary of the stability diagram.

selected, to determine the working point of the ion of interest. Each straight line relies on the DC component used that is a function of $d$. The slope of the straight line, which is the ratio between $U$ and $V$, can be described by the following equation:

$$
\frac{a}{q}=\frac{2 U}{V}=\frac{2 d-1}{2 d(1-d)}
$$

Figure 2 shows several $a / q$ lines obtained using different duty cycle values for the trapping rectangular wave- 
form: the gray parts represent the region of stability for a given duty cycle.

With respect to sinusoidal waveform-driven ion traps in which superimposition of the DC potential on the main trapping field requires the use of an additional DC power supply, the DC component in the DIT can be readily generated by variation of the rectangular waveform duty cycle. This can be easily achieved by varying the parameters entered into the control software of the mass spectrometer.

To experimentally determine the boundaries of the stability diagram, the Mathieu parameters $a$ and $q$ of an ion are chosen such that its working point is located close to the boundary of the stability diagram. In the experiment a duty cycle of $0.5 \%$ is used for ion introduction and cooling. Subsequently, the duty cycle corresponding to the value theoretically predicted for crossing the boundary of the first stability region is used. If the $a$ and $q$ working points calculated for that specific duty cycle are correct, the ion of interest will be lost from the ion trap and no signal is recorded in the mass spectrum. As shown in Figure 3 (lower spectrum), when the monoisotopic ion approaches the working point in the vicinity of the instability region, ion ejection occurs and the corresponding ion signal is detected with lower abundance than the ${ }^{13} \mathrm{C}_{1}$-containing ions, which fall within the stable region of the stability diagram. This abnormality in the isotopic cluster pat- tern is a clear indication that the working point of the monoisotopic ion lies on the $\beta_{r}$ and $\beta_{z}$ boundary lines of the stability diagram. This approach is used to collect several $a, q$ points for $[\mathrm{M}+\mathrm{H}]^{+}$bradykinin fragment $1-5$ $(\mathrm{m} / \mathrm{z} 572)$ and $[\mathrm{M}+\mathrm{H}]^{+}$leukine enkephalin $(\mathrm{m} / \mathrm{z} 556)$, respectively (Figures 4 and 5). However, for the peptide ions used in this investigation, when a large DC component is used (see $-a / q$ quadrant), the graphs obtained from empirical data differ from those theoretically calculated. For duty cycles corresponding to values $q>$ 0.9 and $a>0.6$ the energy imparted to the ion is so high that the ion behavior in proximity to the stability boundary differs from the theoretical ones due to resonant excitation/ejection. For instance, in the case of leukine enkephalin when the $q$ value measured at the boundary line is 0.938 , the difference between experimental and theoretical values is 0.02 . When bradykinin is analyzed, the measured value at 0.964 differs for 0.05 from the $q$ value predicted by simulations. In fact, regardless of the duration of the cooling time the application of a DC component brings ions into a region of the ion trap in which the RF field is larger, accelerating the ions to higher kinetic energies. As an effect of this, ions undergo energetic collisions with the background gas and depletion of the monoisotopic ion is observed due to unwanted fragmentation. Closer examination of the MS spectrum indicates the presence of product ions produced by collisions with the buffer gas

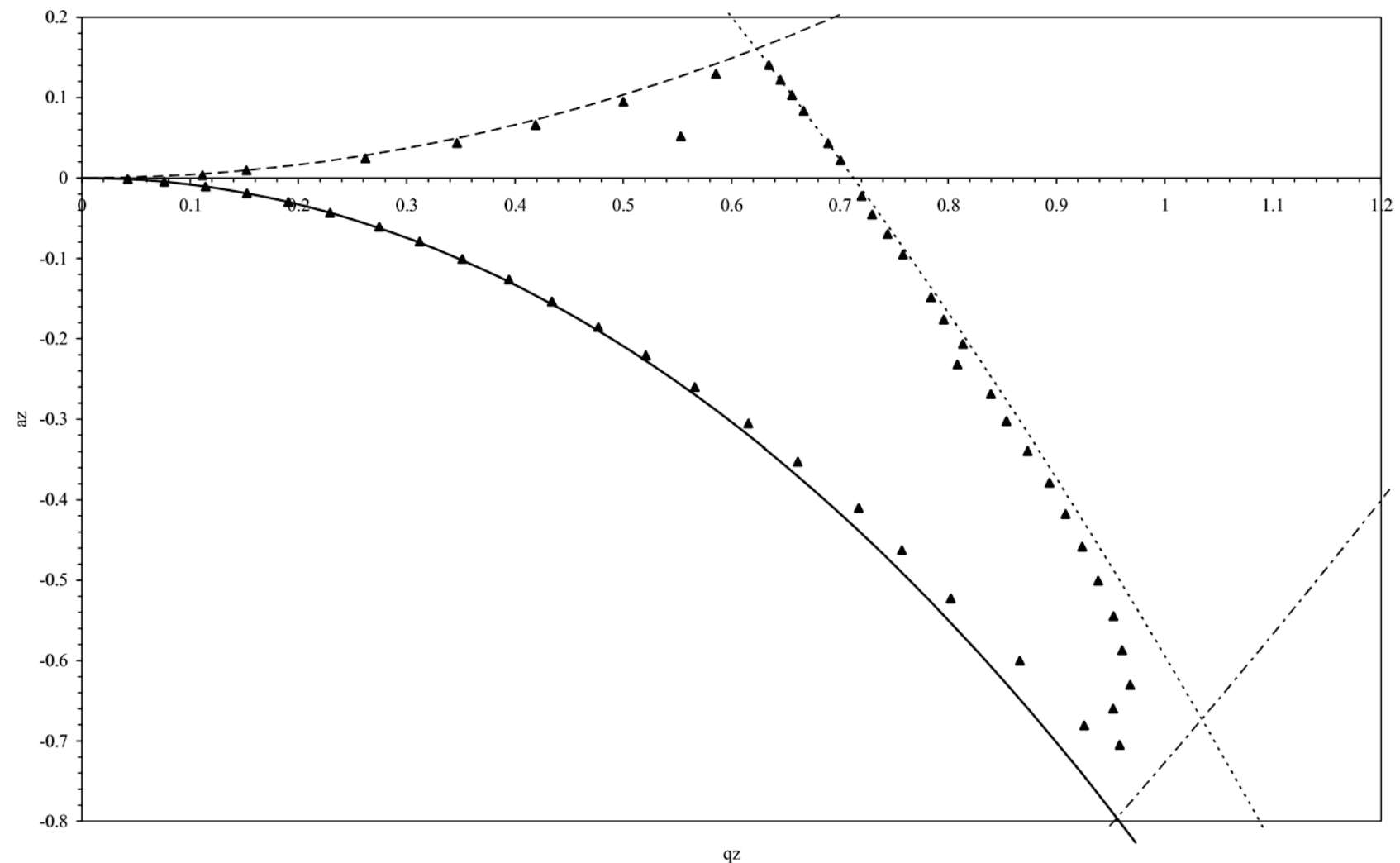

Figure 4. Stability diagram denoting $\beta_{r}$ and $\beta_{z}$ instability boundaries obtained using leukine enkephalin $[\mathrm{M}+\mathrm{H}]^{+}$. 


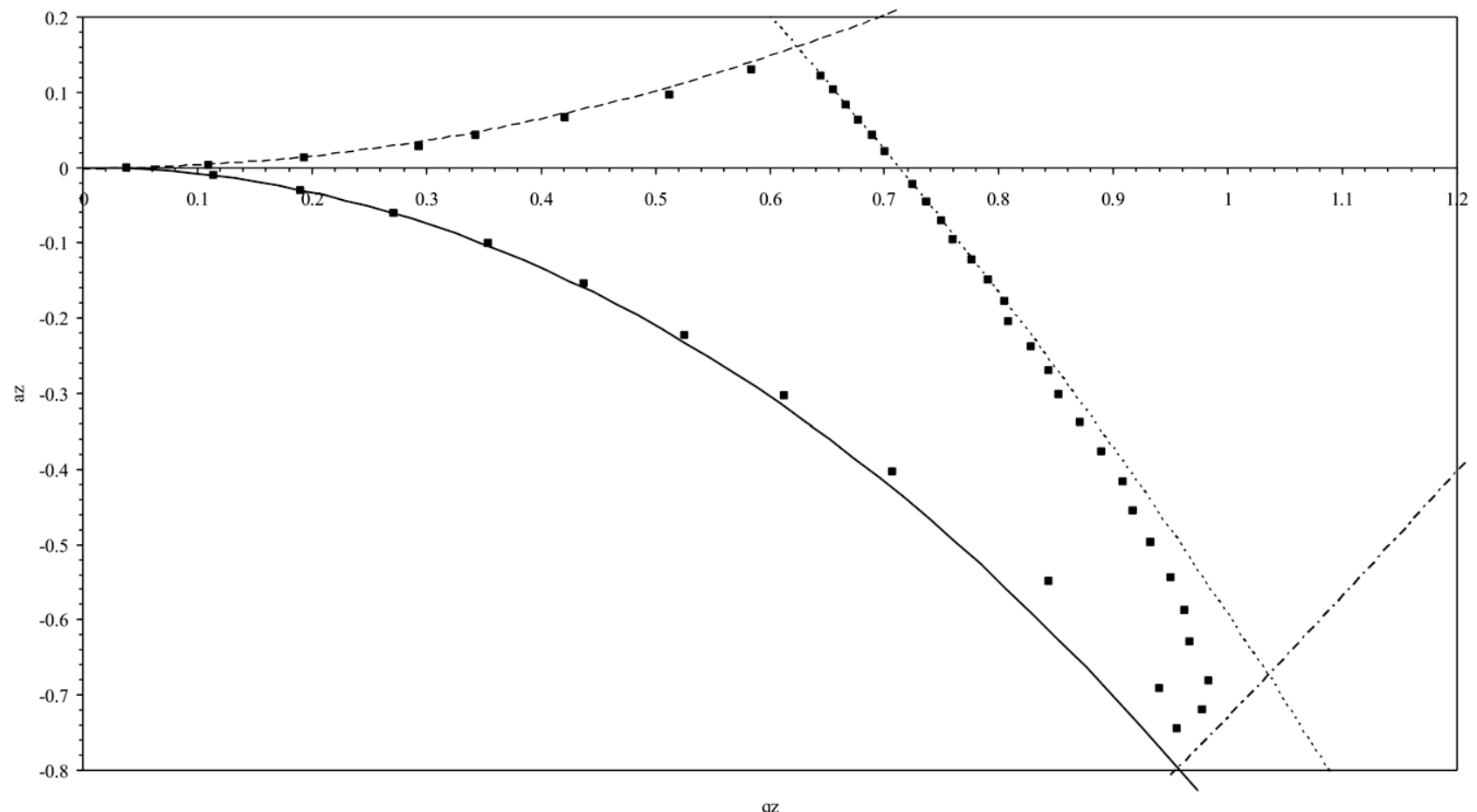

Figure 5. Stability diagram obtained using bradykinin fragment $1-5[\mathrm{M}+\mathrm{H}]^{+}$.

(data not shown). The results presented here suggest that variation of the waveform duty cycle achieved at the software level entering different values in the scan table can result in a more straightforward approach for generating boundary activated decomposition (BAD) without the necessity of an additional power supplier.

\section{Conclusion}

In a digital ion trap the $\beta_{r}$ and $\beta_{z}$ boundary lines of the stability diagram are experimentally determined using leukine enkephalin and bradykinin fragment 1-5. The innovative approach used here shows that manipulation of the waveform duty cycle at the software level allows introduction of a precisely defined DC quadrupole component into the main trapping field without the need of any hardware modification. The boundary lines corresponding to $a$ values up to -0.5 agree with the $\beta_{r}$ and $\beta_{z}$ lines determined theoretically. However, when a large DC component is used $(q>0.9$ and $a>$ $0.6)$ the graphs obtained empirically differ from those calculated by simulations. These findings are in accordance with results previously published for amplitudedriven ion traps. In fact, a similar comparison conducted in a stretched ion trap indicates that large differences between experimental stability diagrams and theoretical approximations are observed in proximity of the $\beta_{z}=1$ boundary line when a large DC component is used [14].

\section{Acknowledgments}

The authors gratefully acknowledge the financial support from Shimadzu Corporation in funding this work. The also thank
Sergey Smirnov for the continuous effort in development of the DIT software.

\section{References}

1. March, R. E. An Introduction to Quadrupole Ion Trap Mass Spectrometry Mass Spectrometry. J. Mass Spectrom. 1997, 32, 351-369.

2. March, R. E.; Todd, J. F. J. Quadrupole Ion Trap Mass Spectrometry, 2nd Edition; Wiley: Hoboken, NJ, 2005.

3. March, R. E.; Todd, J. F. J., editors. Practical Aspects of Ion Trap Mass Spectrometry: Fundamentals (Modern Mass Spectrometry Series, Vol. 1); CRC Press: Boca Raton, FL, 1997

4. Cox, K. A.; Cleven, C. D.; Cooks, R. G. Mass Shifts and Local Space Charge Effects Observed in the Quadrupole Ion Trap at Higher Resolution. Int. I. Mass Spectrom. 1995, 144, 47-65.

5. March, R. E.; McMahon, A. W.; Londry, F. A.; Alfred, R. L.; Todd, J. F. J.; Vedel, F. Resonant Excitation of Ions Stored in a Quadrupole Ion Trap. 1. A Simulation Study. Int. J. Mass Spectrom. Ion Process. 1989, 95, 119-156.

6. March, R. E.; McMahon, A. W.; Allison, E. T.; Londry, F. A.; Alfred, R. L.; Todd, J. F. J.; Vedel, F. Resonant Excitation of Ions Stored in a Quadrupole Ion Trap. 2. Further Simulation Studies. Int. J. Mass Spectrom. Ion Process. 1990, 99, 109-124.

7. Williams, J. D.; Cox, K. A.; Cooks, R. G.; Kaiser, R. E.; Schwartz, J. C. High Mass-Resolution Using a Quadrupole Ion-Trap Mass-Spectrometer. Rapid Commun. Mass Spectrom. 1991, 5, 327-329.

8. Ding, L.; Sudakov, M.; Kumashiro, S. A Simulation Study of the Digital Ion Trap Mass Spectrometer. Int. J. Mass Spectrom. 2002, 221, 117-138.

9. Ding, L.; Kumashiro, S. Ion Motion in the Rectangular Wave Quadrupole Field and Digital Operation Mode of a Quadrupole Ion Trap Mass Spectrometer. Rapid Commun. Mass Spectrom. 2006, 20, 3-8.

10. Ding, L.; Sudakov, M.; Brancia, F. L.; Giles, R.; Kumashiro, S. A Digital Ion Trap Mass Spectrometer Coupled with Atmospheric Pressure Ion Sources. J. Mass Spectrom. 2004, 39, 471-484.

11. Brancia, F. L.; Giles, R.; Ding, L. Effect of Reverse Scan on Mass Measurement Accuracy in an Ion Trap Mass Spectrometer. J. Mass Spectrom. 2004, 39, 702-704.

12. Ding, L.; Brancia, F. L. Electron Capture Dissociation in a Digital Ion Trap Mass Spectrometer. Anal. Chem. 2006, 78, 1995-2000.

13. Konenkov, N. V.; Sudakov, M.; Douglas, D. J. Matrix Methods for the Calculation of Stability Diagrams in Quadrupole Mass Spectrometry. J. Am. Soc. Mass Spectrom. 2002, 13, 597-613.

14. Johnson, J. D.; Peddeer, R. E.; Yost, R. A.; March, R. E. The Stretched Quadrupole Ion Trap: Implications for the Mathieu $a_{u}$ and $q_{u}$ Parameters and Experimental Mapping of the Stability Diagram. Rapid Commun. Mass Spectrom. 1992, 12, 760-764. 